\title{
Consciousness as a new form of the matter's state
}

\author{
Igor Maydykovskiy, \\ "World Public Lab" Private Research Laboratory (Lithuania) \\ e-mail: biveja@gmail.com
}

\begin{abstract}
The article discusses the physical model of the implicative form of Consciousness in the form of a holographic wave matrix, for which the material basis is directly the phase environment that fills the entire Space. It is shown that a similar form of Consciousness that exists outside the human brain can be represented as a kind of software shell that controls all forms of matter by implementing a fractal cyclic iterative algorithm. The condition for the completion of each iterative cycle at each scale level is the observance of the laws of symmetry that ensure the survival of the object in the process of copying-incarnation.
\end{abstract}

Key Words: implicative form of Consciousness, phase medium, holographic wave matrix

I saw and knew that the Cosmos is not dead matter but a living Presence, that the soul of man is immortal, that the universe is so built and ordered that without any peradventure all things work together for the good of each and all. (Richard Maurice Bucke, 1837 - 1902) [1]

\section{Introduction}

Consciousness belongs to one of those concepts that is not included in the topics of research of the physical direction. The hypotheses about the existence of a hidden (implicative) form of consciousness, proposed at different times by such well-known theoretical physicists as David Boom or Roger Penrose, did not aim to propose a physical model that would explain the interaction of matter and Consciousness; and this is quite natural, because according to the official paradigm, space is filled with emptiness, which, of course, does not contribute to the development of an appropriate theory that could connect the form of consciousness with the presence of a material environment.

Therefore, here we can speak not so much about not a desire as about the inability of physical science to admit that Consciousness can have a material basis. For this reason, none of the physicists asks the question, like, - How does matter "know" what it should be? What makes an electron be an electron, water - water, a human - a human, etc.?

Indeed, from the point of view of scientists, if we assume that "something" governs the forms of matter, then it will be necessary to recognize science as a section of theology, and all forms of matter as a manifestation of divine power. Therefore, the disputes about, - By whom and how was the world in which we live was created? - They will remain groundless exactly until a person realizes that Consciousness and Matter have a single structural basis. In this sense, we can assume that the desire to understand, - What is wrong in physics? - Did not arise out of nowhere and not only in connection with a lack of understanding of the physical mechanism of the interaction of Consciousness with physical reality. There are enough reasons to doubt the correctness of the classical Paradigm.

All rights reserved (C) When copying the entire text or its individual parts, a link to the original is required 
After all, not a single physicist, for example, can explain what force makes the Earth revolve endlessly around the Sun, and electrons around the nucleus of an atom. However, it is one thing when such doubts are born as a result of an informational occasion, and a completely different case, when, due to some circumstances, a person himself has to directly face inexplicable physical phenomena or artifacts.

In such situations, when a person is not able to understand the physics of a phenomenon, he becomes a kind of experimental subject in an experiment conducted by Nature herself, inviting the person himself to find the correct answer. In this sense, the reason for the appearance of this article is just a certain Artifact, the properties of which again forced us to think about the correctness of the classical paradigm. In this sense, looking ahead, it will not be an exaggeration to assert that the new paradigm based on the developed Theory of the Phase Medium allows in its applications to explain both the very possibility of the existence of such Artifacts and its properties.

\section{Artifact}

An artifact is usually called an object that has characteristics and properties that have no analogues in our World, and which cannot be described within the existing paradigm. In this case, we are talking about the "black stone of Knowledge", the unusual properties of which and its appearance on Earth cannot be explained except by the existence of a supercivilization on our planet more than 5 thousand years ago. It was at such a period of time that the Keeper of the "stone", under whose patronage he is, indicated, and which provided scientists with the opportunity to investigate the Artifact in one of the physics institutes. These studies have raised new questions that still remain unanswered. Outwardly, the "stone" resembled a large chicken egg, had a warm to the touch, matte smooth dark gray surface, similar to ceramics, and was clearly of man-made origin. As the Keeper of the Artifact said, he received the box with the "stone" several years ago from the hands of the previous Keeper, who left this World.

The box contained a scroll with a text, as it turned out later, written in biblical Hebrew, as well as a list of all former keepers, from which it followed that this Artifact has been with the keepers for at least more than two millennia. According to the text, the "black stone of Knowledge" is one of the three "stones" left on Earth by certain Overlords.

In one of the many legends about King Solomon (994 - 930 BC) it was possible to find information that the "black stone of Knowledge" together with another, "red stone of Justice", was transferred into the possession of King Solomon at the beginning of his reign (973 - 933 BC). That is, it happened more than three millennia ago. Today, the "red stone" can be found on the territory of modern Israel.

The text also speaks of the third, "the white stone of Energy". As the researchers suggest, as well as according to the guardian, the "stone of Energy" is located somewhere in the mountains of Tibet in a monastery or underground Temple; the "white stone" is closed from the eyes, as the one who looks at it can instantly go blind.

The text did not mention the reasons for the appearance of these "stones", as well as the purpose, their location on Earth, however, it says that the stones (brothers in the text) must meet. Therefore, without delving into historical facts and evidence in one way or another connected with the existence of the Artifact on Earth, one should mention one ancient Tibetan legend, which indicates a moment in the history of humankind when all three "brothers" should meet: 
... when all Truth in the outer World is lost due to wars, thirst for power and lucre, then a dynasty of enlightened Overlords will come with a "Great Army" to defeat the forces of evil and establish the Golden Age. Under their enlightened rule, the Earth will finally become a place of peace and abundance and be filled with the jewels of Wisdom and Compassion.

According to the author, all three "stones": Justice, Knowledge and Energy, called "brothers" - these are the three brothers, called the warriors of the "Great Army". Apparently, the names of the "stones" also have a huge meaning. In the absence of Justice on Earth, humanity will not receive either Knowledge or Energy, otherwise it can destroy itself. Therefore, the unification of stones is both a modus and a pathway, allowing without causing harm to humanity, to fill the World with Justice, the Wisdom of Love and Compassion!

Some facts that the author came across personally indicate that the Energy and Knowledge contained in the "stones", and which a person can acquire with their help, cannot be used for selfish purposes. Anyone who decides to use the Knowledge and the Power of the "stones" for evil will always be stopped or even destroyed before he can carry out his plans.

If we talk about the results of studies of the physical properties of the "black stone", then the impossibility of interpreting these results, focusing on ideas about the properties of matter within the framework of the existing paradigm, is striking.

Here are some facts from reports on the results of studies that were carried out in one of the physics institutes using modern equipment, and which can expand our knowledge and understanding of the forms of existence of matter, at the same time suggesting directions for future research:

- The Artifact was an object in the form of an elongated ellipsoid of revolution (elongated spheroid) with the dimensions of the golden section: $71 \mathrm{~mm} \times 44 \mathrm{~mm}$. The smooth, matte surface in dark gray was reminiscent of ceramic.

- The material from which the Artifact was made turned out to be harder than diamond. During the tests, the diamond head of the measuring device crumbled into dust, and a scratch remained on the surface of the "stone", which disappeared the next day.

- The experiment with a powerful laser puzzled scientists, since a laser beam with a power of about $5 \mathrm{~kW}$, having come into contact with the surface of the stone, disappeared, as if passing an invisible border, found itself in infinite space, while the temperature of the surface of the stone practically did not change. The backward scattering cone from the point of contact of the beam with the surface of the "stone" resembled a shock wave; the scattering power was insignificant in comparison with the power of the laser itself. That is, it can be assumed that the Artifact either "absorbed" all five kilowatts of power, or destroyed the energy of the laser beam, or the Artifact itself was a portal of transition to another dimension.

- One of the experiments consisted in scanning the "stone" with a system of low-power lasers, as is done when recording a hologram. After processing the information on the monitor screen around the "stone", we saw a three-dimensional picture of spherical field structures nested one into another, like in a nesting doll, which slowly rotated relative to each other. The diameter of the largest sphere was about $60 \mathrm{~cm}$. The "images" on the spheres resembled a multitude of translucent "living" color holograms flowing into one another.

Obviously, the shape of the Artifact in the form of an ellipsoid of revolution was not chosen at random and performs an important function. 
Since it is known that, the surface of an ellipsoid of revolution is a locus of points in space, for which the sum of the distances to each of the two foci is a constant value. Moreover, if we imagine that the inner surface is a mirror, then the radiation energy emanating from one of the foci of the ellipsoid, after reflection, will be collected in another focus. Therefore, we can say that the "stone" is a concentrator of the energy of external oscillations of longitudinal waves. At the same time, the energy circulating inside the ellipsoid will be, as it were, sealed in its structures.

However, perhaps the most important experiment was carried out in a sense in a random way, but its result is the most informative in terms of demonstrating the capabilities of the Artifact. At the time of the research, the Keeper of the "black stone" voluntarily handed it over to a confidant who agreed to coordinate the course of the research. One of the employees of the office, where the "stone" was kept in the safe, decided to secretly take the Artifact over the weekend in order to show it to his close relatives. Here is an approximate sequence of facts and events that speak of the ability of the "black stone" to meaningfully change physical reality, influencing both the consciousness and behavior of a person, and the state of aggregation of the mother and the surrounding space.

According to an office employee, on the way to the dacha, the following happened: On the highway, somewhere in the middle of the road, in front of the car, "Something" suddenly appeared in the form of a certain transparent substance, similar to the movement of warm air, reminiscent of a small tornado.

The car flew into this "Something".., after which the jeep spun, turned over a couple of times, hit the ground several times and carried it to the side of the road. After a special vehicle delivered the jeep and its owner to the dacha, this "something" appeared again.

Now THIS hovered over the house, and after a while, the whole house at once flared up by itself, like a match. At this time, according to the victim, a blue-white pulsating glow in the form of a halo appeared around the "stone", which disappeared as soon as the flame engulfed the entire house.

There were several more episodes associated with the manifestation of the unusual properties of the Artifact; and all of them were associated with a situation when someone, without the consent of the Keeper, wanted to take advantage of the "stone" in their own interests. For example, at the initiative of the research coordinator, without the consent of the Keeper, an attempt was made to place the Artifact in another office; and after the Artifact was in the safe in a new place, all computers stopped working in this office. However, as soon as the "stone" was returned to its original place, the work of computers was restored by itself.

Based on the results of the research carried out, as well as comparing a number of historical facts presented in the materials of independent researchers, an important conclusion can be drawn:

- The Artifact is part of an ambitious plan to preserve earthly civilization. The need to preserve Knowledge and the subsequent revival of civilization could be associated with the inevitability of a natural cataclysm, which was known in advance, and as a result of which life on Earth had to be interrupted and then revived. Suffice it to recall the "World Flood", which took place about 6 thousand years ago.

For the preservation and protection of Knowledge, a "black stone" was created, which contains all the information about the structure of the Universe, technologies and the organization of the management structure of the earth's civilization. 
This Knowledge would allow humanity at the right moment after the catastrophe to return to the composition of developed civilizations inhabiting the Universe. This may not be the only scenario for the development of the alleged events. There is another version, about the presence of aliens on Earth and about the alien origin of the Artifact. In this case, it can be assumed that the consequences of the catastrophe were so significant that only after thousands of years, the Earth was able to recover, and humanity got the chance and opportunity to use this "Treasure".

It is important to note that the Artifact demonstrates the possibility of direct interaction of the human brain with a certain hidden form of Consciousness, existing in the form of a "living" structured material substance capable of controlling energy flows. Moreover, the very process of interaction from the side of the "hidden form" is based on the Algorithm of the creative thought process. The work of this algorithm allows you to scan human consciousness, fixing a person's intentions, taking into account the slightest deviations in his mood and behavior. The algorithm assumes enumeration of options and the choice of a method and instrument for influencing the situation, guided by the principles of Goodness and Justice.

Such "behavior" of the Artifact indicates the presence of a built-in Algorithm of a reasonable creative Beginning, which can be interpreted as a manifestation of the implicative (hidden) form of the higher cosmic Reason. This "Reason", existing outside the human brain and interacting with the human consciousness, is able to directly change the state of physical reality, using an unknown form of energy and physical mechanisms that allow it to control material objects.

That is, we can conclude that the Artifact, demonstrating the inextricable connection between consciousness and matter and manifesting the creative Beginning, reproduces a natural functional mechanism, of which it is a man-made analogue. And here there can be no doubt that this is exactly how Nature is arranged. In this sense, based on the fact of the existence of an Artifact with fixed unusual properties and respecting people's belief in the existence of God, we have to substantiate and prove that the "creative Beginning", declared by theologians as the prerogative of the Creator, can be represented in the form of a transparent, physically grounded model.

Therefore, our further research aims to offer a theoretically grounded physical model that will allow us to understand and explain the existence of two related forms of Consciousness. On the one hand, this is a hidden form of a certain distributed structured network, similar in its properties to a neural network, and on the other hand, it is a physical mechanism that is capable, interacting with human consciousness, directly changing physical reality.

\section{Consciousness as a factor indicating the existence of a new state of matter}

The simplest example proving the existence of a natural mechanism, in which consciousness and matter are combined in one physical process, is the process of birth of an electron-positron pair from a vacuum. As you know, outer space is permeated by streams of cosmic particles and ionizing gamma radiation. The source of gamma quanta is not only the centers of galaxies and stars, such as our Sun, but also space itself, in which the continuous process of birth and annihilation of positrons and electrons takes place.

Let us ask ourselves a question - How does a future electron at the time of its birth learn about the characteristics and parameters that it should have? What controls the state of the surrounding space at this moment in time, when the energy of a gamma quantum is transformed into the energy of matter in the form of two particles? 
The very fact of the existence of this process has been confirmed experimentally, although, in contrast to the natural process, the conduct of experiments that make it possible to record the creation of an electron-positron pair from a vacuum in laboratory conditions is associated with the need to create high gradients of field structures. These can be constant and homogeneous mutually perpendicular electric and magnetic fields, or strong laser fields. As an example, we can refer to the work devoted to studies related to the problem of studying the creation of an electron-positron pair $\left(\mathbf{e}^{-} \mathbf{e}^{+}\right)$from a vacuum irradiated in combination with strong laser and Coulomb fields. [2] That is, in this experiment, the energy of a gamma quantum is instantly transformed into the energy of matter in the form of two particles with opposite charge signs.

By the way, within the framework of the classical theory, there is no physically grounded model that explains the nature of the charge, as well as the nature of the electric field, which has been assigned the status of a "special type of matter". Obviously, in such a situation, it is impossible, within the framework of the official paradigm, to explain that the process of the creation of an electron-positron pair is controlled by a certain structure endowed with the ability of a creative Beginning.

Therefore, the author, as an alternative, developed and proposed the Theory of the phase Medium, based on the recognition of the materiality of the structure of Space. [3] This allows us to consider any physical process that forms reality as a result of the work of a natural physical mechanism that unites consciousness and matter.

It is important to note that from the point of view of the physical process, the operation of the mechanism of projection and materialization of an object does not depend on its size. Therefore, the results of studies presented below within the framework of the Phase Medium Theory can be equally extended both to the process of electron birth and to the process of birth of planets and Galaxies. However, before, on the basis of the results of theoretical research, present the substantiation of the materiality of the implicative (hidden) form of Consciousness, it is necessary to understand - what we know about Consciousness.

Consciousness as a concept has a wide range of interpretations and is the subject of research in philosophy, sociology, psychology, as well as disciplines that study the problems of artificial intelligence. Of particular practical interest are studies of the borderline state of consciousness, when its presence or absence can be determined as a result of testing the brain activity of people in a coma or in a state of clinical death.

Consciousness is associated with a person's ability to reproduce reality as a projection of all external and internal connections that determine the essence of any body, matter or process.

Consciousness simultaneously reflects and forms the world of physical reality, therefore the creative process, that is, a person's ability to create and change physical reality, is the main form of manifestation of consciousness.

It is the creative, evolutionary type of thinking that can be considered the main advantage of "Homo sapiens". However, Homo sapiens himself is a creation of Nature, and in this sense, the creative Beginning given to him by Nature is only an element of a more significant and deeper (hidden) functional natural mechanism, which we have already mentioned.

An integral element of the cognitive process is the process of accumulating and preserving knowledge. In the world of physical reality, this function, in addition to the human brain itself, can be performed by material carriers capable of restoring a picture of reality at the level of an explicit form of consciousness. 
In this sense, the implicative (hidden) form of consciousness should also be endowed with a certain protected functional mechanism that would ensure the process of accumulating and storing information directly related to human thinking activity.

Therefore, before talking about the possibility of the existence of consciousness outside the human brain, it is necessary to understand and explain the physical mechanism that supports the function of the creative Beginning in Nature, that is, everything that is included in the concept of "the Creator". In other words, it is necessary to find a certain higher implicative form of Consciousness and a material carrier corresponding to this form, which, through an explicit form of consciousness connected with the human brain, is able to cognize the world, create and change physical Reality.

In this case, it should be assumed that the explicit form of consciousness, directly connected with the human brain, is endowed with a kind of interface, which in its essence has a similar physical and functional basis with the implicative (hidden) form. It is also obvious that, taking into account the two-way feedback, the process of modifying the forms of consciousness should be of a cyclical quantum character.

This, in turn, requires synchronization of the process of interaction between explicit and latent forms of consciousness at the structural level, and also requires compliance with the principle of equivalence of phase and time characteristics. That is, within the framework of the model under consideration, each of the forms of consciousness can exist as one of the phases of a continuous cyclic process.

Summarizing all that has been said about the properties of consciousness, its implicative form can be conventionally represented as a hierarchical structure, highlighting three conventional levels in it, namely:

The object level, which is directly connected with the human brain and provides the function of dynamic interaction with physical reality through a sensory mechanism and has interface elements for communication with the latent form of consciousness.

The system level, which through the interface maintains communication with the object level and implements several algorithms, - it's an algorithm for projection and interaction of levels, an algorithm for storing information, as well as an algorithm for managing an aggregate of projections.

The highest program level, which determines the order of interaction of all levels of consciousness and controls all functional connections of all structured objects of the Universe from elementary particles to planetary systems and Galaxies.

Functionally, the implicative form of consciousness can be divided into its own wave matrix and its projection, which directly controls the explicit form of consciousness. Thus, both forms of the wave matrix, being united by a cyclic process, can function independently of the physical shell in the form of a human brain. In this sense, the two-way continuous exchange process between the projection of the wave matrix and the matrix itself in the structure of the implicative form cannot be interrupted even if the physical functions of the brain are "disabled".

In other words, the sensory function of the brain can be identified with the work of an "analog computer", which in the structure of the object form of consciousness can be considered autonomous to a large extent. Therefore, in the case of "turning off" this "computer", the human consciousness in the form of a projection of the wave matrix will reflect the state of the same matrix in the structure of the implicative form, continuing to participate in the cyclic process. 
Everyone who is familiar with a computer device usually considers the implementation of the algorithm of the software shell as a process that does not have a common structural basis with the hardware and exists in a certain virtual space in the form of interaction of mathematical operators in the structure of certain algorithms. However, in relation to the implicative form of consciousness, the use of the new concept of the wave matrix, as an analogue of the program shell, allows us to consider the structural basis of the implicative form as a new state of matter.

\section{The Material World - from the Hologram}

One of the first to propose the concept of the structural basis of physical reality, taking into account the built-in programmed wave matrix, was the eminent English physicist of the 20th century David Joseph Bohm. According to his hypothesis, the Universe is a giant fluid Hologram or "holomovement" in which the World exists simultaneously in two realities - it is the material world itself and a certain structure that defines the general order, which exists in some implicit form in each area of spatial-temporal Continuum.

What is the essence of this not accepted and obscure concept by David Bohm, moreover, greatly damaging his impeccable reputation as a theoretical physicist at that time? [4] According to Bohm himself, the described order can be a projection of higher volumetric levels of reality, in which the apparent stability and hardness of objects and creatures is created and maintained thanks to the continuous process of clotting - deployment. In this process, subatomic particles belonging to object reality are constantly "clotting" into a hidden order, and then recrystallization (restored).

According to David Bohm's ideas, it is consciousness that lies outside the physical level of reality and, existing in a deep implicative (hidden) form, determines the general Order and controls other forms of matter. From the point of view of a materialist, the metaphysical nature of such an assumption is obvious, therefore, in the scientific world, his hypothesis was dubbed as difficult to understand and not having practical application. However, the paradox of the situation lies in the fact that David Bohm himself, while remaining an adherent of Einstein's model of the structure of the Universe, believed that the process of cyclical "erosion" of matter into a hidden order (clotting) and subsequent restoration (deployment) of the object proceed in absolute emptiness without material intermediaries.

Obviously, excluding the physical environment and replacing it with such an indefinite concept as "subatomic particles", it was impossible to understand the mechanism and substantiate the process of clotting - deployment itself. Despite this, the hypothesis of David Bohm, the essence of which neither his colleagues nor the scientific community as a whole could understand and appreciate, remains relevant today, as it creates the preconditions for revising the accepted official paradigm. And the first step in this direction is the recognition of the materiality of the phase Medium, that is, the recognition of a new form of matter as the basis of the structure of space.

Independently of David Bohm, the famous neurosurgeon Pribram Karl H. proposed the idea of using the holographic principle to explain the phenomena associated with the mental activity of the brain. After conducting many operations and experiments, he came to the conclusion that human memory is separated from the thinking process associated with the sensory function of the brain. He drew his conclusions on the basis of comparing the velocity of the mechanisms of thinking and memory with the reaction time during synaptic transmission of impulses along the nerve fiber. [5] Such velocity when transferring, memorizing and retrieving unlimited amounts of information from memory can be carried out only at the field level. 
This conclusion was made by Pribram Karl H. in the mid-1960s, when he began to consider the brain as a projection of a hologram. As a result of acquaintance with the holographic theory, it turned out that it allows one to explain many phenomena and facts in the activity of the brain, reveals many neurophysiological mysteries that scientists of that time were unsuccessfully struggling with.

For example, the ability to instantly recognize a familiar face after many years, even if the appearance of the acquaintance has changed "beyond recognition." In a word, the famous neurophysiologist, professor at Stanford University Pribram Karl H., becoming an adherent of the holographic theory, proposed a model of the distributed structure of human memory, when each part of the brain copies and repeats the contents of the whole.

Just like a piece of a holographic wave matrix contains information that can be used to reconstruct a whole image. This important property of the holographic wave matrix allows us to say that the control wave matrix of the human brain is built according to the same physical laws as the wave matrix of any material object. Therefore, the hierarchical structure of consciousness can be viewed as an implicative form of a general higher order that determines the structure of any kind of matter.

Another important aspect of the scientific revolution made by Bohm is that, according to his hypothesis, the physical reality surrounding us, in fact, exists as a materialized projection of the Hologram and is a spatially quantized dynamically balanced structure. In other words, both forms of physical reality - that is, the implicative form that determines the general order and manifested form, that is, all bodies, objects and processes - consist of the same elements of an elastic and homogeneous phase medium that has a regular structure. Accordingly, the form of existence of physical reality of any scale level and of any nature is the materialized projection of the wave holographic matrix in the cyclic process of copying-incarnation in the structure of the phase environment. (Here, the term: copying-incarnation according to the Phase Medium Theory is analogous to the term: clotting - deployment, proposed by David Bohm).

To designate the functional features of each of the three levels of the implicative form of consciousness, the diagram (Fig. 1) shows the structure and algorithm of the interaction of the implicative form of consciousness with physical reality in the cyclical process of copyingincarnation.

The first thing to pay attention to is the structure of velocity rates in exchange processes. Such a distribution of velocity can be explained within the mathematical model of an automatic control system with a multi-loop feedback system. It is known that for the stable operation of such systems, the speed of information flow in the feedback loop must always exceed the velocity of the process itself. This over velocity ultimately determines the stability of both any element and the System as a whole.

That is, if the maximum velocity with which a certain process can proceed at the object level is equal to the velocity of light $c^{*}$, then the velocity of the energy-information flow in the feedback loop of the same process, taking into account the phase delay, cannot be less than $\sim c^{2}$, otherwise as a result accumulation of mismatch errors, the process will simply "fall apart".

It is obvious that the beginning of the phase of "object incarnation" can begin only after the completion of the process of modifying the wave matrix. That is, the cycle period and the phase delay at the object level will be determined by the inertial properties of the phase medium and the information transfer velocity in the feedback loop, which in turn depends on the velocity of the wave matrix modification process. 


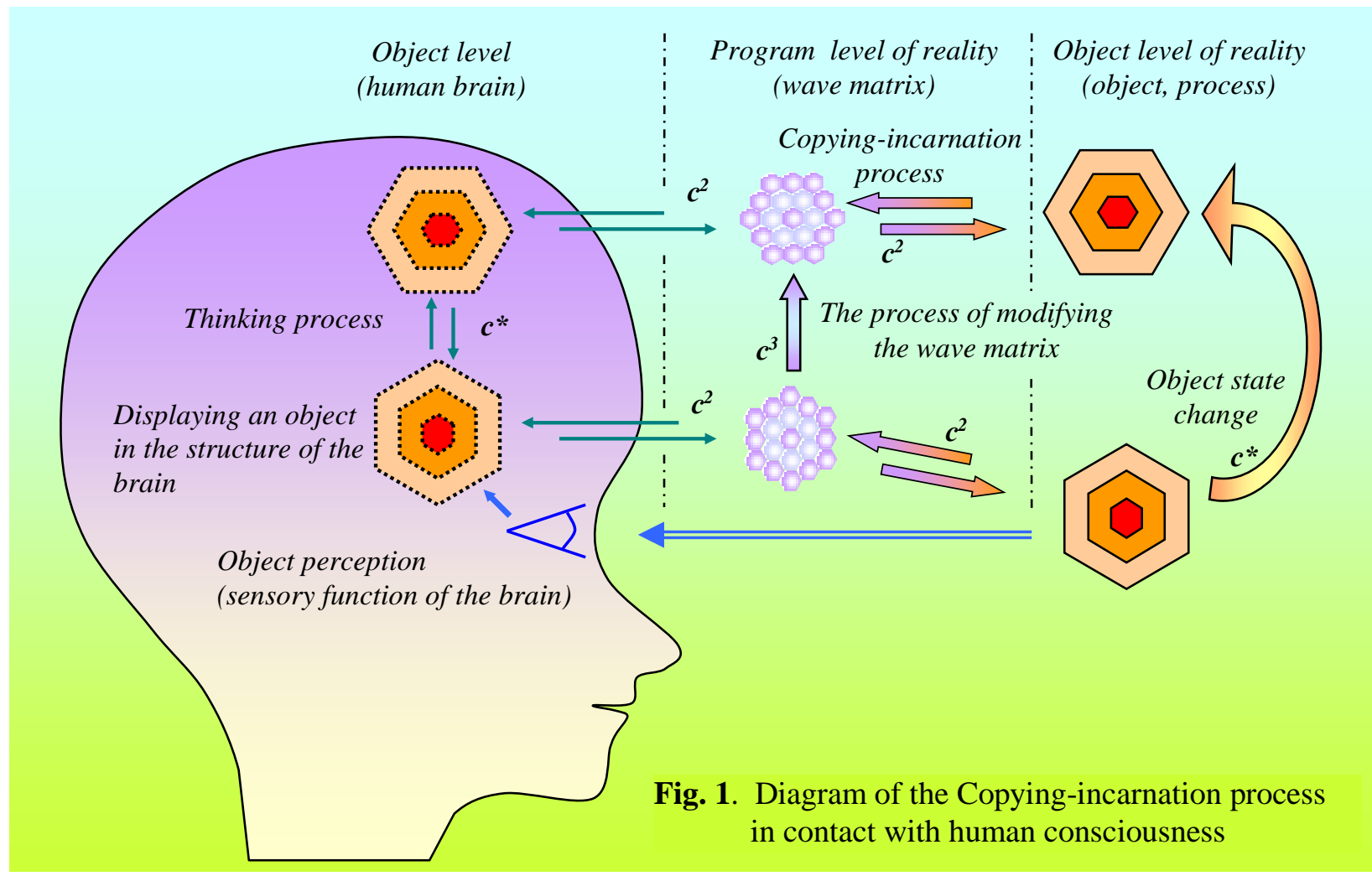

Within the framework of the model under consideration, the feedback loop for the object level, both from the side of the human brain and from the side of physical reality, should be considered the chain of the copy- incarnation process. In turn, the copying-incarnation process itself also has its own feedback loop, due to which the modification of the wave matrix is provided. Accordingly, the velocity of the process in the matrix modification loop must be at least $\boldsymbol{c}^{\mathbf{3}}$, that is, the entire process of materialization of the wave matrix proceeds, as it were, in two stages and is covered by two loops of the feedback system. (A more detailed physical model of the copying-incarnation process is considered in the author's work at the link indicated above.) [3]

\section{Physical Model of Consciousness}

The model of the structure of the phase environment proposed in [3], as well as the considered mechanism and algorithm for the interaction of the implicative form of consciousness with physical reality in the cyclic process of copying-incarnation, make it possible to formulate a new concept such as a physical model of Consciousness. This concept covers the whole range of previously existing hypotheses and assumptions about the ways, forms and mechanisms of the interaction of Consciousness with physical reality and allows us to assume that Consciousness has a material basis. Therefore, before we can determine the content of the new concept and the limits of applicability of the proposed model, it is necessary to solve the main task - to prove that the specified mechanism functions not only at the level: a physical reality - the human brain, but is also an operating mechanism participating in the implementation of the "creative Beginning" and defining the essence of this concept.

In other words, it is necessary to establish "who" acts as the "main prompter", which indicates, What characteristics and parameters the electron should have at the moment of its birth?

Unlike David Bohm, the famous physicist Benoit B. Mandelbrot did not offer revolutionary hypotheses about the structure of the Universe, his approach was to study the structures inherent in living nature. His fundamental work "The fractal geometry of Nature" [6] was, in a sense, a good experimental confirmation of the theory of fractals developed later. 
The mathematical algorithm proposed by Benoit Mandelbrot, being transferred to a physical model, makes it possible not only to explain the natural mechanism of the implementation of the holographic principle, but also to endow this functional mechanism with the ability to create reality. That is, it allows explaining the ability of Nature to "consciously" reproduce (clone) the structure and characteristics of an "object", creating any number of clones at each scale level of object reality. The main feature of the algorithm is that it is always formalized in the form of a system of equations, which allows using the iterative method to achieve the desired result. And if for modern computers this results in a huge expenditure of time and resources, then the cosmic Reason carries out this process in each cycle of copying-incarnation, thus presenting Nature as the best designer, ideal builder and engineer.

The third participant who offered the evidence base for our research can be considered Gennady. S. Melnikov, who was one of the first to see the connection between the fractal structure of Nature and its ability to create order out of chaos. Melnikov proved that by combining Euler's theory of harmonic series and Mandelbrot's theory of fractals, any space-time structure can be represented as a system of equations for the geometric field of spatial frequencies, that is, as a holographic wave matrix (tensor) that governs of the structure and shape of matter. [7]

Thus, Melnikov substantiated the holographic principle of organizing the structure of space, from the point of view of a mathematical model, thereby confirming Boom's hypothesis. In other words, Melnikov's theory allows us to consider the holographic wave matrix, combined with the Mandelbrot algorithm, as a functional basis of the hidden order, and as a material form of implicative consciousness.

In accordance with the theory of G. Melnikov and the presented classification of spatial configurations and cyclic structures, a very unexpected but unique conclusion can be made:

- Of all the possible combinations, the Mega System, being based on resonant structures obeying the laws of random and thinned harmonic series, can itself reproduce any spatial and spatial-temporal object with a probability of unity by implementing a fractal cyclic iterative algorithm with the exclusion off one or more components of a harmonic random series.

In this case, the algorithm for making a decision to exclude one or another harmonic, being embedded in a multi-loop network structure of feedbacks, monitors in each cycle at each scale level the observance of the laws of symmetry that ensure the survival of the object in the process of executing the iterative algorithm. In this sense, in accordance with the presented scheme of the algorithm (Fig. 1) and taking into account the velocity rates of exchange processes in the feedback circuits, during one cycle of copying-incarnation, each object will be checked for compliance with the laws of symmetry $10^{8}$ times.

Thus, the Program of the Fractal Cyclic Iterative Algorithm (FCIA) built into the cyclic process of projection of the wave matrix allows us to consider this process responsible for the control function of all forms in the hierarchical structure of matter.

Accordingly, this algorithm, being built into the implicative (hidden) form of Consciousness, is the "main prompter" or element of the cosmic Reason, which indicates - What characteristics and parameters the electron should have at the moment of its birth. In such a situation, it is impossible not to touch upon the topic: How this World was created, and whether this process had a Beginning. 


\section{The Birth of the Universe, Beginning}

If we came to the conclusion that the cosmic Mind exists, then the question immediately arises - Has it always been so? And if not, what could be the Beginning, when and for what reason did it happen? An attempt to find an answer to these questions immediately runs into the definition of the initial conditions. And here we again return to the Theory of the phase medium and Melnikov's theory, which proposes to consider all spatial and space-time configurations, structures and cyclic processes as existing in the multidimensional phase space in the form of concentric spherical shells, each of which is locally orthogonal to each of the three spatial coordinates. Thus, Melnikov's theory proves the existence of a regular, elastic phase medium in which all configurations, processes and ordered structures, being objects of space-time, have a fractal nature. Therefore, in such a model, the phase medium filling all infinite space has always existed.

At the same time, Melnikov's theory, combining the theory of Euler's harmonic series, Mandelbrot's theory of fractals and the holographic principle proposed by David Bohm, creates the conditions and prerequisites for the formation of a new Paradigm that explains, among other things, the origin of the Universe. That, given the material form of Consciousness, allows us to consider the proposed structure and configuration of space as the initial conditions for the emergence of the cosmic Reason, - Let's try to figure it out - How could this happen?

The primordial state of the Universe cannot be considered chaos, as the ancient philosophers suggested and as modern physicists - materialists do it, suggesting to consider the "Big Bang Theory" as the primary cause of Everything. The Big Bang theory is based on the same chaos theory, and therefore it can be said as if it takes roots from ancient Greek philosophy. The debate about the correctness of this model continues today, and according to the well-known theoretical physicist Stephen W. Hawking, - Nobody can say what happened before the Big Bang.

Within the framework of the new paradigm, the wave matrix, that is, the geometric field of spatial frequencies, is described by a system of equations in a two-dimensional frequency plane. In this case, based on the proofs of the famous theoretical physicist Paul Ehrenfest, we can conclude that chaos in such a space is, in principle, impossible, as well as random nonharmonic processes. The whole space is filled with the Harmony of oscillations of longitudinal spherical waves, where quasiperiodic processes can also exist, representing complex combinations like single waves or solitons. Therefore, in accordance with the principles of conformal projections, any material object, being a projection of the wave matrix, will represent a harmonized structure, physically consisting of the same structural elements of the phase medium as the wave matrix itself.

And if at the large-scale level of the Universe and the structures of Galaxies it can be assumed that the Cosmic Intelligence is in a state of "search" for forms, which for an external observer is represented as random processes of transformation of matter and energy, then at the level of our physical reality the World is more determined - and the electron will no longer be any different. In this sense, any matter consisting of atoms and elementary particles is also structurally internally harmonized.

Then it is not clear what nuclear - physicists study when they on charged particle accelerator "break" one harmonized structure with the help of another similar structure, assuming that the resulting fragments are the components of the "particle" they are studying. These "shards" in the form of socalled subatomic particles or quarks are considered structureless, point objects. 
Or, in other words, in the opinion of physicists, protons and other baryonic particles are, as it were, composed of these indefinable point objects, of which, in turn, the overwhelming part of the substance we observe is built. For example, it is believed that a proton consists of three quarks and due to the small size of the latter, most of the space inside the proton is filled with emptiness!

Why complicate the model so much, because it is easier to assume that elementary particles created by Nature in the process of evolution are self-sufficient objects of the microworld and do not need to be divided into parts. And this self-sufficiency is explained, figuratively speaking, quite simply You cannot play a melody on a musical instrument that can reproduce only one note! And this note, called a quark, does not fit into the general "melody" of the holographic wave matrix in any way.

Returning to the question of the "Beginning", it can be argued that before the appearance of what can be called the Universe today, there was a primitive Order. That is, a certain ordered regular distribution of energy in space, where there is no, and cannot be, time. On the one hand, this state can be defined as a state of uncertainty (singularity), which, from the point of view of an external observer, can last as long as desired. On the other hand, having defined this state as a primitive order in timeless space, that is, in the absence of any matter and concentrated Energy, it is necessary to admit the possibility of fluctuation of this state. For example, as a result of random synchronization of oscillations of a certain number of primitive cells of the phase medium. The transition to a new state can hardly be called an "explosion", but THIS can be called the Beginning with confidence!

The origin of Consciousness and Matter at the level of primitive order occurred simultaneously, starting with elementary particles, in accordance with the already considered fractal cyclic iterative algorithm and with the rapid transition of this process to higher scale levels in terms of the complexity of objects. At the same time, there was a transition of the structure of space from a state of primitive order to a structured phase environment. A further scenario based on self-organization of mutually conditioned structures was a harmonic process that, with the passage of Time, sought to occupy more and more space.

Simultaneously with the development of the process of the birth of the Universe, the feedback system was also improved, using at each subsequent scale level more and more perfect mechanisms and tools to create conditions that ensure its own survival. In this sense, the generation of the structure of the cosmic Reason and the subsequent reproduction of a more subtle instrument in the form of human Consciousness with the function of the creative principle fully corresponds to the solution of the same single task associated with ensuring the survival of the already born Universe!

The famous American scientist, philosopher and inventor of the 20th century Richard Buckminster Fuller spoke very accurately about the role of man and humanity in this process, - Human wealth his intellect and skill increase with their use; and this human craving for creation is explained by the need to regulate the entropy of the Universe. Richard B. Fuller believed that man carries the function of a refined anti-entropy. That is, in this case, Human Consciousness acts as a kind of functional of a natural mechanism, in a system of feedbacks, which also ensure the survival of the Universe.

In this sense, it should be noted that the destructive paradigm in which Mankind exists today fundamentally contradicts the law of the existence of cosmic Reason on planet Earth, as one of the Centers with an anti-entropic function in our Universe. That is, ANY actions of people, initially leading to destruction "inside" of Civilization, contradict the fundamental law of the Universe. 
Obviously, taking into account the inertial properties of the phase medium, there is a physical limitation of the size of the Universe, which is determined by the permissible number of spectral ranges and the maximum oscillation frequency of longitudinal spherical waves in the structure of an element of the phase medium, that is, limited by the size of the Wigner-Seitz cell and the Planck wavelength, that is, by the size of a quantum of matter.

According to preliminary calculations, at present the process of the development of the Universe has ended, and has passed into a state of a stationary oscillatory mode of changing the density of the phase medium with phases: compression - expansion. In this case, the dimensions of the Universe will be determined by the period of the second harmonic in the structure of the spherical shell, which separates the Universe from the infinite space, which is in a state of primitive order. The duration of this period is $\mathbf{1 0 . 8 4}$ billion years. According to the latest data, the age of the Universe is $\mathbf{1 3 . 4}$ billion years. Accordingly, the Universe is now at least at the beginning of the second evolutionary Cycle of its development in the initial stage of the expansion phase.

Some scientists, remaining "captives" of the Big Bang theory, believe that the recognition of the cyclical nature of the process does not exclude the compression of space again to a Singularity point and the subsequent new a "Big Bang". In this case, it would be necessary to recognize the transition of all the Energy of the fundamental harmonic in the structure of longitudinal spherical waves into a state of singularity, that is, to recognize the possibility of the death of the Universe. Which is definitely out of the question, since an elastic structured medium, having once emerged, cannot disappear, destroying the harmonic process due to which it exists, which directly contradicts the law of survival of the Universe, as well as the law of conservation of Energy, which in the new paradigm has a slightly different meaning. Since our Universe is only half of the Mega System; in another part of this system, the electron is replaced by a positron.

Now we can answer the question that served as the reason for our research - Does Consciousness survive after irreversible bodily death? Obviously, the content of the answer is a natural inference from our research:

- Consciousness in the form of a projection of the wave matrix of a single individual, devoid of an "Interface" as a result of bodily death, remains in the structure of the implicative form in the form of a certain functional appendage of the root wave matrix. At the same time, the information content of this matrix, accumulated in the process of an individual's life, does not change, like its status. Undoubtedly, the possibility of establishing direct contact with the root wave matrix remains through a virtual Interface using the properties of the phase medium.

In a broader context, human Consciousness has always been and remains a part of the all-embracing cosmic Reason, therefore, it cannot disappear even in the event of bodily death, that is, "shutdown" of the physical functions of the human brain.

\section{Conclusion}

The unity of Consciousness and Matter - it is the structural and functional basis of the Creative Principle in Nature. As the highest level of the hidden order, this state of the phase environment can be considered the "driver" of all processes in the world of physical reality, including the very birth of the Universe. Or, to put it in simple language - the physical essence of a hidden form of a higher order, uniting consciousness and matter - this is the invisible Creator of our World in which we live. 
Therefore, I would like to draw attention to the fact of attempts to create artificial intelligence, making a person an appendage of the digital mind. The digital environment can destroy the very "interface" that is in every person and thanks to which a person has the ability to communicate with the cosmic Reason. Intellect exists and surrounds us, and a person is a part of it, that is, a part of the cosmic Reason, due to which human abilities are unlimited. Therefore, if we assume that man is a subtle instrument in the system of feedbacks that regulate processes in the Universe, then a reasonable question arises, - How long will Nature "tolerate" the actions of man, in his desire to build the tower of Babel and thereby try to "replace" this working mechanism with a digital analogue. In this case, of course, we are not talking about areas related to the robotization of technological production or the use of robots in logistics and transport navigation systems.

You should not strive to become God - you just need to be able to negotiate with him, using what is already in each of us. After all, today the main task of a person is to understand the mechanism of communication with the cosmic Reason, and thanks to this, learn to apply his own limitless possibilities and abilities that are given to a person from birth. This is one of the main tasks of man and his mission in the Universe!

\section{References}

1. Bucke Richard Maurice: Cosmic Consciousness: A Study in the Evolution of the Human Mind. // INNES \& SONS, 1311 Sansom Street, Philadelphia, 1905 Edition.

https://www.pdfdrive.com/cosmic-consciousness-a-study-in-the-evolution-of-the-human-mind$\underline{\mathrm{e} 175987400 . \mathrm{html}}$

2. A. I. Milstein et al.: Polarization-Operator Approach to Electron-Positron Pair Production in Combined Laser and Coulomb Fields. // Physical Review A - Atomic, Molecular, and Optical Physics, vol. 73, no. 6, pp. 062106-1 - 062106-10, American Physical Society (APS), Jun 2006.

3. Igor Maydykovskiy, Petras Užpelkis: Energy Matter Space Time. // Collection of articles published in the "Intellectual Archive" journal, 2020 - 2021, pp. 64, Upload all Articles in one file: https://b43d4e5a-fd6d-4f8d-a5de-

5a92c850b1b6.filesusr.com/ugd/c514bf_c161f7fc6ce441d582cada9fdefc793a.pdf

4. David J. Bohm: Wholeness and the Implicate Order. // London: Routledge, 1999, pp. $186 f$

5. Pribram Karl H.: The Corsini Encyclopedia of Psychology and Behavioral Science. // Volume 3 /

Third Edition, W. Edward Craighead, Charles B. Nemeroff - Wiley, 2002 - pp.1241-1952

6. Benoit B. Mandelbrot. W. H. Freeman and Co.: The fractal geometry of Nature. // San Francisco, 1982, pp. 460.

7. Gennady S. Melnikov: Space-time fractal unity // LAP LAMBERT Academic Publishing, Oct. 2014. 